Stefan Kubica, Hagen Ringshausen, Jörg Reiff-Stephan, Marius Schlingelhoff (Hrsg.) 1. Automobil Symposium Wildau: Tagungsband Technische Hochschule Wildau 2016
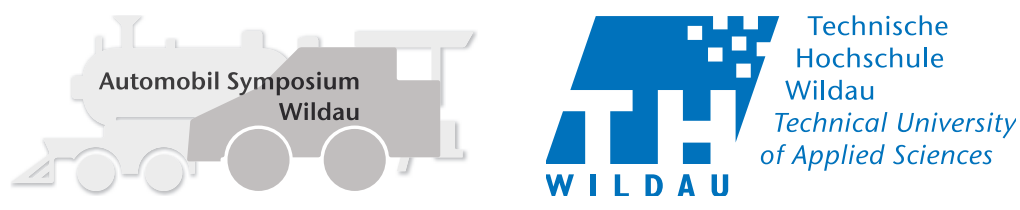

\title{
Automatische Kalibrierung eines Kamerasystems zur Verkehrsdatenerfassung auf Basis von Navigationssensorik
}

\author{
Sebastian Schulz, Daniel Wagenknecht
}

\section{Zusammenfassung}

Die visuelle Erkennung von Verkehrsteilnehmern mittels Bildverarbeitung wird zunehmend wichtiger. Bisher wurde die exakte äußere Orientierung des Kamerasystems durch eine photogrammetrische Kalibrierung mit genau verorteten Passpunkten erreicht. Selbstkalibrierende Kamerasysteme, die Navigations-Sensorik zur Orientierungsbestimmung nutzen, haben einige Vorteile und können gerade in mobilen Anwendungsbereichen neue Türen öffnen. Gibt es die Möglichkeit, ein Kamerasystem für die Verkehrsdatengewinnung zu entwickeln, das handelsübliche Sensortechnologie verwendet und dennoch eine ähnliche Genauigkeit erreicht wie die klassische Kalibrierung? Zur Bestimmung der äußeren Orientierung kommen eine GigE-SmartCam, ein Raspberry Pl, eine inertiale Messeinheit (Inertial Measurement Unit - IMU) und ein GPS-Empfänger zum Einsatz. Die IMU bestimmt die Orientierung der Kamera und der GPS-Empfänger die Position und Höhe. Die Daten werden zusammen mit den Bilddaten der Kamera an einen bildverarbeitenden Server übermittelt. Die Genauigkeit der Hoch- und Rechtswerte des GPS-Empfängers entsprechen den technischen Angaben. Die gemessene Höhe hat eine starke Abweichung zur photogrammetrisch berechneten Höhe. Diese Abweichung hat einen großen Einfluss auf die Genauigkeit der Kalibrierung. Die Orientierungswerte der IMU entsprechen der erwarteten Genauigkeit. Das System arbeitet zuverlässig und die automatische Kalibrierung ist technisch möglich.

\section{Einleitung}

\subsection{Motivation}

Die automatisierte Erfassung von Verkehrsteilnehmern ist im Verkehrsmanagement eine wichtige Aufgabe. Das Wissen über die Anzahl, Position und Fahrtrichtung der Verkehrsteilnehmer ermöglicht die Berechnung verschiedener Kenngrößen, wie zum Beispiel des Verkehrsflusses, der Vorhersage über das Verhalten in bestimmten Verkehrssituationen, der Verkehrsdichte zu einer bestimmten Uhrzeit usw. Im Bereich der Fahrzeugerfassung wurden bisher viele verschiedene, zuverlässige Sensorkonzepte entwickelt. Diese variieren in ihren Vor- und Nachteilen. Seit in den vergangenen Jahren die doi: 10.15771/ASW_2016_5

Rechenleistung von Computern stark zugenommen hat, wurde auch die digitale Bildverarbeitung zur Fahrzeugerfassung genutzt. Im Vergleich zu anderen Sensoren, sind Kameras preiswerte, mobile Sensoren, die einen geringeren Aufwand beim Aufbau haben. Zusätzlich kann mit innen eine großflächige und weiträumige Erfassung mehrerer Verkehrsteilnehmer sowie die Analyse derer Fahrtverläufe realisiert werden.

Neben der Abhängigkeit von Kamerasystemen von wechselnden Witterungs- und Beleuchtungsbedingungen wird insbesondere deren mobile Nutzung durch die erforderliche manuelle Kalibrierung erschwert, um Bildinformationen in ein übergeordnetes Koordinatensystem zu übertragen. Eine große Herausforderung ist die Bestimmung der äußeren Orientierung der Kamera. Dazu gehören ihre Position (Hochwert X, Rechtswert Y, Höhe Z) im Raum und ihre Blickrichtung (Kurswinkel $\Psi$, Neigungswinkel $\theta$, Rollwinkel $\phi)$. Diese Parameter sind notwendig für die Startwerte des späteren Matching-Verfahrens. Dabei werden das Kamerabild und ein Orthobild, anhand von Straßenmarkierungen und Geoformen übereinandergelegt. Umso genauer die Startwerte sind, desto besser funktioniert das Matching-Verfahren. Ein möglicher Ansatz zum Bestimmen der Parameter ist das photogrammetrische Verfahren. Dabei werden dreidimensionale Referenzpunkte im Erfassungsbereich der Kamera verteilt und mittels eines GNSS vermessen. Die bildliche Repräsentation dieser Messpunkte wird ebenfalls bestimmt. Über das Wissen um die Position eines Referenzpunktes in Bild und Welt kann die äußere Orientierung der Kamera nun bestimmt werden. Die Umsetzung dieser Art der Kalibrierung ist sehr zeitintensiv und in einigen Situationen sogar sehr schwierig.

Dieser Artikel beschreibt eine Methode zur automatisierten Bestimmung der äußeren Orientierung basierend auf einer Inertial Measurement Unit (IMU) und einem GNSS-Empfänger (Global Navigation Satellite System). Die ermittelten Werte dienen als sog. Startwerte für das spätere Matching der aufgenommenen Bilder auf ein orthorektifiziertes Kartenbild.

1.2 Voraussetzungen für die automatische Kalibrierung

Das System zur automatischen Kalibrierung der äußeren Orientierung unterliegt bestimmten Voraussetzungen. 
Zum einen benötigt das System ein GNSS-Signal. Das bedeutet, dass das System in geschlossenen Räumen, ohne »Sichtkontakt« zu den GNSS-Satelliten, nicht funktioniert. Des Weiteren ist das System darauf angewiesen, dass die IMU ordnungsgemäß funktioniert. Bei fehlenden Informationen über die Blickrichtung oder die Position der Kamera, kann die Berechnung zur äußeren Orientierung nicht ausgeführt werden. Vor einer Kalibrierung müssen die Werte der IMU und des GPS-Empfängers auf Plausibilität geprüft werden.

\subsection{Stand der Technik}

Die automatische Bestimmung der äußeren Orientierung wird bereits in Smartphones genutzt, ein Beispiel ist die Google Sky Map (Google 2016). Bei dieser App werden ebenfalls das GNSS und die integrierte IMU des Telefons genutzt, um die Blickrichtung und Position der Kamera zu bestimmen. Hält man sein Smartphone in Richtung Firmament, kann man in der App ablesen, welche Sterne und Sternbilder man in Blickrichtung sehen kann. Nachteil dieser Anwendung ist, dass die Geschwindigkeit und die Genauigkeit der berechneten Orientierung nicht ausreichen, um daraus zuverlässige Georeferenzierungen im urbanen Bereich vorzunehmen. Herkömmliche GNSS-Empfänger, wie sie in Smartphones verbaut sind, bieten nur eine Update-Rate von $1 \mathrm{~Hz}$ (Flax 2016). Für die automatische Kalibrierung im verkehrstechnischen Bereich ist eine Update-Rate von $20 \mathrm{~Hz}$ vorgesehen. Dies entspricht der Bildrate der eingesetzten Kamera.

Des Weiteren werden solche Systeme bereits genutzt, um Georeferenzdaten für Kartenbilder aufzunehmen. Das System befindet sich dabei in einem Flugzeug. Ebenfalls über ein GNSS und eine IMU werden Position und Blickrichtung der Kamera während des Fluges gemessen. Beispiele für solche Systeme sind ANTAR und ARGOS vom DLR (Kozempel 2012). Die Berechnung der orthorektifizierten Bilder anhand der Mess- und Bilddaten erfolgt dann nachträglich. Nachteil dieses Systems sind seine Größe und der hohe Preis (Grenzdörfer \& Zuev 2007).

\section{Herangehensweise zur automatischen Kalibrierung}

Die Bestimmung der äußeren Orientierung des Kamerasystems basiert auf einem Navigations-Sensorsystem. Dafür werden grundsätzlich ein Kamera- und ein Sensorsystem, sowie eine Recheneinheit benötigt. Abhängig von der Leistungsfähigkeit der Systemzusammenstellung sind eine oder mehrere Software-Anwendungen erforderlich. Dazu gehören die Sensordatengewinnung, die Prozessierung der Messwerte, das Integrieren in die Bilddaten und die Übertragung an den weiterverarbeitenden Server.

Digitale Messkameras eignen sich für das vorgestellte Konzept besonders, da sie eine langanhaltende Stabilität der inneren Orientierung aufweisen (Wagenknecht 2013). In erster Linie wird die Kamera für die Bildgewin- nung genutzt. Qualitativ hochwertige Kameras verfügen zusätzlich über einfach nutzbare Recheneinheiten,

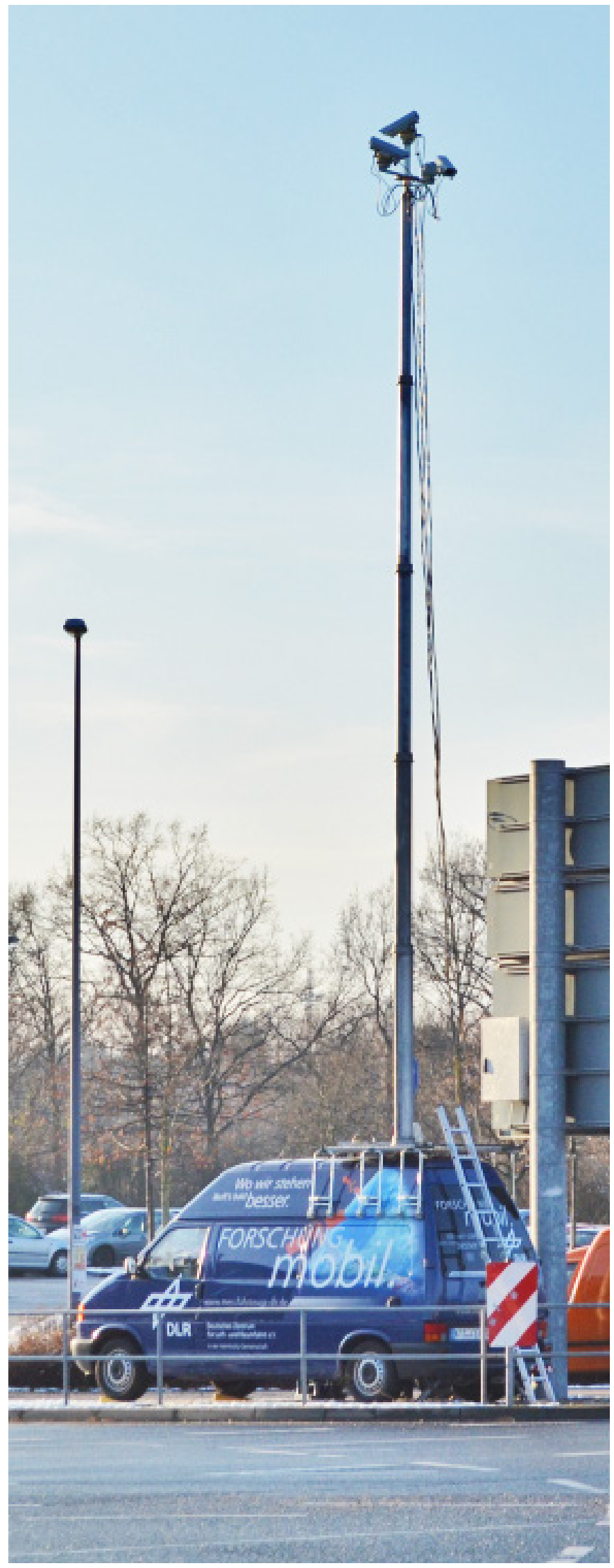

Abb. 1 UTRaCar mit ausgefahrenem 12-Meter-Teleskopmast in Dresden 2012 (Quelle: eigene Aufnahme).

die die Funktion der Kamera individualisieren. Beispielsweise könnte das Integrieren der Orientierungswerte in 
die Kameradaten oder das Übertragen der Gesamtdaten an den Server durch eine geeignete Kamera erfolgen. Digitale Messkameras mit ausreichender Rechenleistung könnten eine weitere Recheneinheit unnötig machen. Neben der nötigen Rechenleistung ist eine zuverlässige und schnelle Netzwerkverbindung wichtig. Bei unkomprimierten Bildern von etwa 1 Megabyte (MB) (monochrom) und einer Bildwiederholrate von 20 Bildern pro Sekunde (engl. frames per second - fps) ergibt sich eine erforderliche Bandbreite von $20 \mathrm{MB} / \mathrm{s}$. Bei einer Farbkamera verdreifacht sich dieser Wert in etwa durch die drei Farbkanäle Rot, Grün und Blau.

Die Blickrichtung und Rotation der Kamera bezogen auf das Weltkoordinatensystem wird durch die IMU bestimmt. Konkret wurde der Typ Flyduino 9DOF benutzt. Für gewöhnlich benötigt eine IMU eine Initialisierung bei bekannter Lage und Ausrichtung. In Kombination mit einem 3-Achsen-Magnetometer entfällt dieser Schritt, und die Messeinheit kalibriert sich bei jeder neuen Position von selbst.

Die Position der Kamera im Weltkoordinatensystem wird über den GNSS-Empfänger bestimmt, wobei hier der Typ Venus638FLPx benutzt wurde. Beide Sensoren, IMU und GNSS bestimmen ihre Daten bezogen auf ihr eigenes Koordinatensystem. In einem initialen Kalibrierungsprozess müssen diese Koordinatensysteme mit dem der Kamera zusammen bestimmt werden. Dafür wird die äußere Orientierung der Kamera mit Hilfe der Navigationssensorik und simultan dazu über das photogrammetrische Verfahren bestimmt. Über die photogrammetrisch berechnete Orientierung der Kamera kann nun die Navigationssensorik kalibriert werden.

Wenn der Funktionsumfang, bzw. die Rechenleistung der Kamera nicht ausreicht, um die Messwerte der $\mathrm{Na}$ vigationssensorik zu ermitteln und zu berechnen, muss eine externe Recheneinheit dazu genommen werden. Die verwendete IMU verwendet den $\mathrm{I}^{2} \mathrm{C}$ Bus (serieller Bus zur Kommunikation zwischen verschiedenen Schaltungsteilen) und das GNSS die serielle UART-Schnittstelle (seriellen Schnittstelle). Die verwendete Kamera von Leutron Vision PicSight P141B-Smart502-AS verfügt nur über eine RS232-Schnittstelle (Leutron Vision 2013). Daher ist eine externe Recheneinheit nötig. Dafür wurde der Einplatinenrechner Raspberry PI Modell B ausgewählt.

\section{Ergebnisse und Auswertung}

Nachdem das photogrammetrische Verfahren verwendet wurde, um die Navigationssensorik zu kalibrieren, wird sie jetzt genutzt, um die Tauglichkeit des Systems zu ermitteln. Dafür wurde an verschiedenen Positionen die Kalibrierung mit eingemessenen Passpunkten durchgeführt. Parallel dazu wurden die Position und Ausrichtung der Kamera über die Navigationssensorik aufgenommen. Im Anschluss wurden die durch die Navigationssensorik gemessenen Daten mit den photogrammetrisch berechneten Daten verglichen.
Für den Testaufbau wurden die IMU, das GNSS und der Raspberry PI in das Kameragehäuse montiert. Das System wurde auf dem $12 \mathrm{~m}$ hohen Teleskopmast des UTRaCar (Urban Traffic Research Car - Messfahrzeug des DLR) befestigt (Abb. 1). Die Passpunkte wurden auf einer ebenen Fläche neben einer schwach frequentierten Straße aufgestellt. Dadurch konnte die Position der auf dem Fahrzeug montierten Kamera geändert werden. Für die photogrammetrische Kalibrierung wurden die Passpunkte mit einem Trimble 5700 Navigationssystem und
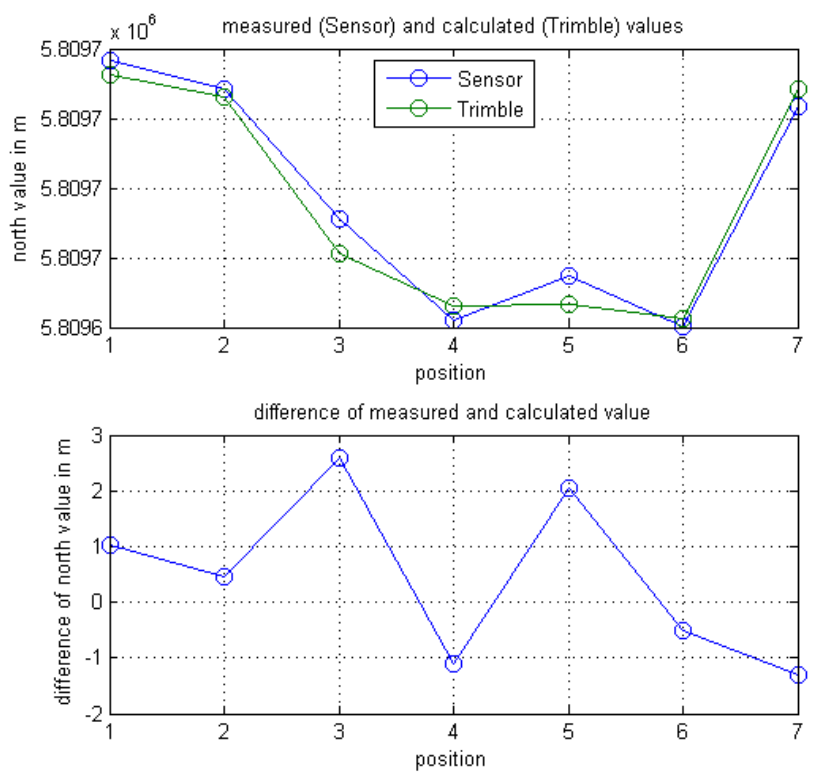

Abb. 2 Hochwert und Differenz zur Referenzmessung für 7 verschiedene Messpositionen, gemessen mit Trimble und berechnet mit Australis gegen den mit GNSS (Sensor) gemessenen Hochwert (Quelle: DLR).
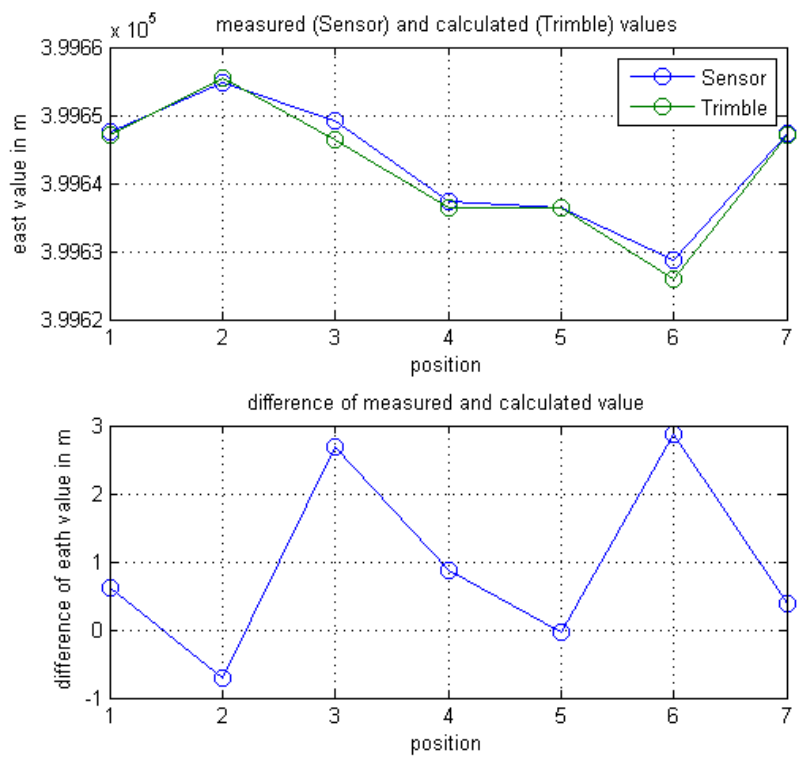

Abb. 3 Rechtswert und Differenz zur Referenzmessung für 7 verschiedene Messpositionen, gemessen mit Trimble und berechnet mit Australis gegen den mit GNSS (Sensor) gemessenen Rechtswert (Quelle: DLR).

den Korrekturdaten des SAPOS-Dienstes (Satellitenpositionierungsdienst der deutschen Landesvermessung) vermessen. Die Kameraposition, sowie die Blickrich- 
tung der Kamera wurden nachträglich mit der Software Australis (Software zum Berechnen der Kameraposition anhand von georeferenzierten Passpunkten im Kamerabild) berechnet. Um die automatische und die photogrammetrische Kalibrierung zu vergleichen, wurden für die ermittelten Werte die Differenz sowie die Standardabweichung berechnet.
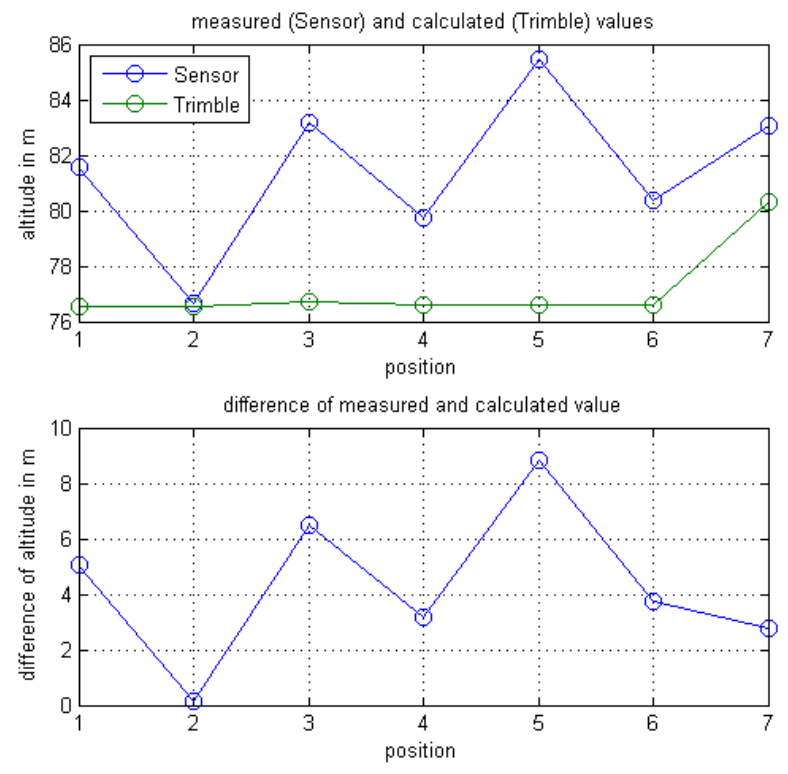

Abb. 4 Höhen und ihre Differenzen für 7 verschiedene Messpositionen, gemessen mit Trimble und berechnet mit Australis gegen die mit GNSS (Sensor) gemessene Höhe (Quelle: DLR).

In Abb. 2 und Abb. 3 sind die Differenzwerte zur berechneten Kameraposition zu sehen. Als Referenz diente dabei immer die von Australis berechnete Kameraposition. Die größten Abweichungen vom Referenzwert im Hoch- zur Hälfte ausgefahren, um den Neigungswinkel der Kamera zu verändern.

Die vom Sensor gemessene Höhe unterliegt einer starken Schwankung. Die größte Abweichung vom Referenzwert beträgt $8,838 \mathrm{~m}$. Die durchschnittliche, absolute Abweichung beträgt 4,311 m und die Standardabweichung 5,032 m. In Tab. 1 sind die Messreihen, die ab-
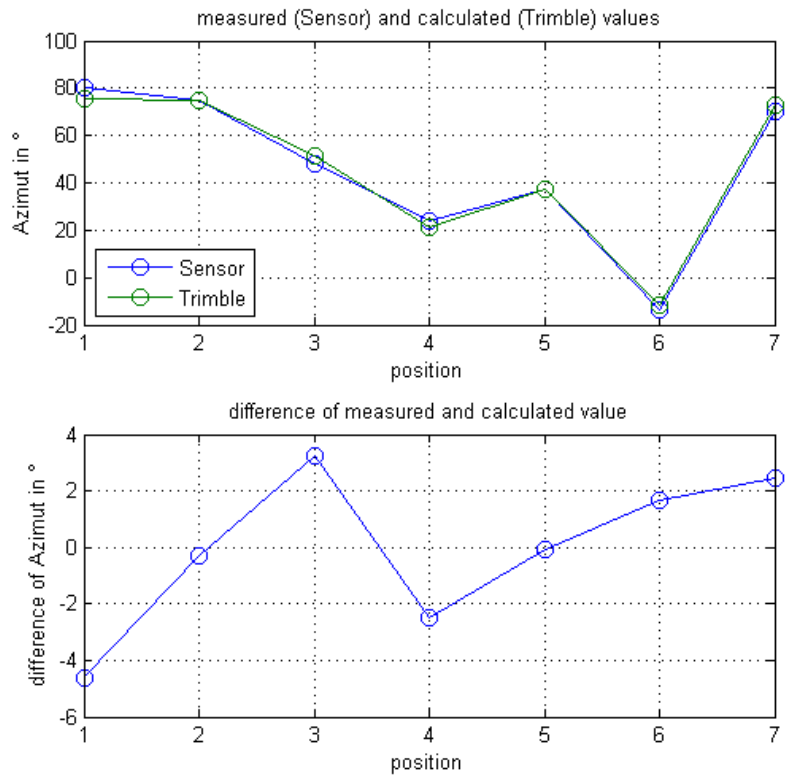

Abb. 5 Azimut und Abweichung für 7 verschiedene Messpositionen, gemessen mit Trimble und berechnet mit Australis gegen den von der IMU bestimmten Kurswinkel (Quelle: DLR).

soluten und die resultierenden mittleren Fehler für jede Raumkomponente $\mathrm{dx}$, dy und $\mathrm{dz}$ und deren Quadrate dargestellt. Darüber hinaus ist die Wurzel des mittleren quadratischen Fehlers (RMS) der quadratischen Anteile

Tab. 1 Differenz und Standardabweichung der gemessenen Position zur berechneten Position

\begin{tabular}{|c|c|c|c|c|c|c|}
\hline & $\mathrm{dx}[\mathrm{m}]$ & $d x^{2}\left[m^{2}\right]$ & dy $[\mathrm{m}]$ & $\mathrm{dy}^{2}\left[\mathrm{~m}^{2}\right]$ & $\mathrm{dz}[\mathrm{m}]$ & $\mathrm{d} z^{2}\left[\mathrm{~m}^{2}\right]$ \\
\hline Messreihe 1 & $-1,023142$ & 1,04681955 & $-0,625496$ & 0,39124525 & $-5,043714$ & 25,4390509 \\
\hline Messreihe 2 & $-0,471547$ & 0,22235657 & 0,711613 & 0,50639306 & $-0,134056$ & 0,01797101 \\
\hline Messreihe 3 & $-2,575369$ & 6,63252549 & $-2,678291$ & 7,17324268 & $-6,468676$ & 41,8437692 \\
\hline Messreihe 4 & 1,104891 & 1,22078412 & $-0,87243$ & 0,7611341 & $-3,177943$ & 10,0993217 \\
\hline Messreihe 5 & $-2,049292$ & 4,1995977 & 0,027977 & 0,00078271 & $-8,838049$ & 78,1111101 \\
\hline Messreihe 6 & 0,5192017 & 0,26957041 & $-2,864465$ & 8,20515974 & $-3,768197$ & 14,1993086 \\
\hline Messreihe 7 & 1,293105 & 1,67212054 & $-0,390904$ & 0,15280594 & $-2,74654$ & 7,54348197 \\
\hline Summe & $9,0365 \mathrm{~m}$ & $15,2637 \mathrm{~m}^{2}$ & $8,1711 \mathrm{~m}$ & $17,1907 \mathrm{~m}^{2}$ & $30,1771 \mathrm{~m}$ & $177,2540 \mathrm{~m}^{2}$ \\
\hline Mittelwert & $1,2909 \mathrm{~m}$ & & $1,1673 \mathrm{~m}$ & & $4,3110 \mathrm{~m}$ & \\
\hline
\end{tabular}

wert liegen bei 2,575 $\mathrm{m}$ und im Rechtswert bei 2,864 $\mathrm{m}$. Die Standardabweichungen liegen bei 1,477 m für Hochund 1,567 m für den Rechtswert. In Abb. 4 ist die gemessene und berechnete Höhe der Kamera zu sehen. Die photogrammetrisch bestimmte Höhe liegt konstant bei $76 \mathrm{~m}$. An Position 7 wurde der Mast des UTRaCar etwa angegeben

Das in Abb. 5 gezeigte Diagramm zeigt die Abweichung der gemessenen Kurswinkel $(\Psi)$ zu den photogrammetrisch berechneten Winkeln. Die größte, absolute Abweichung beträgt $4,598^{\circ}$, die mittlere, absolute Abweichung liegt bei $2,113^{\circ}$ und die Standardabweichung bei $2,582^{\circ}$. In Tab. 2 sind die berechneten Differenzen und 
Standardabweichungen zu sehen. Der Nickwinkel $(\theta)$

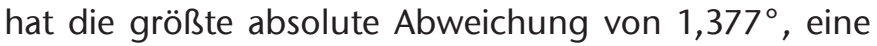
mittlere, absolute Abweichung von $0,681^{\circ}$ und eine Standardabweichung von $0,844^{\circ}$. Der Rollwinkel $(\phi)$ hat die größte, absolute Abweichung von 3,369 ${ }^{\circ}$, eine mittlere, absolute Abweichung von $1,785^{\circ}$ und eine Standardabweichung von $2,033^{\circ}$.

Die mit der Navigationssensorik ermittelten Werte sind ausreichend genau um die Start-werte für das spätere
Hierbei ist hervorzuheben, dass die Rotationsbestimmung anhand der inertialen Sensorik bereits in der Referenzmessung eine Genauigkeit erreichen konnte, die den weiteren Einsatz der IMU rechtfertigt.

Auch die Positionsbestimmung der Kamera mittels des GNSS-Empfängers erbrachte die erwartete Genauigkeit (SkyTraq 2011). In der künftigen Weiterentwicklung des Systems muss hier eine Möglichkeit gefunden werden, die Ergebnisse zu verbessern. Mögliche Verfahren baTab. 2 Differenz und Standardabweichung der gemessenen Rotation zur berechneten Rotation

\begin{tabular}{|c|c|c|c|c|c|c|}
\hline & $\mathrm{d} \Psi\left[^{\circ}\right]$ & $\mathrm{d} \Psi^{2}\left[{ }^{0}{ }^{\circ}\right]$ & $\mathrm{d} \theta\left[{ }^{\circ}\right]$ & $d \theta^{2}\left[{ }^{\circ} 2\right]$ & $\mathrm{d} \phi\left[{ }^{\circ}\right]$ & $\mathrm{d} \phi^{2}\left[{ }^{\circ} 2\right]$ \\
\hline Messreihe 1 & 4,59846 & 21,1458344 & 0,236175 & 0,05577863 & 1,14551 & 1,31219316 \\
\hline Messreihe 2 & 0,294196 & 0,08655129 & $-1,34177$ & 1,80034673 & $-0,283732$ & 0,08050385 \\
\hline Messreihe 3 & $-3,23434$ & 10,4609552 & 0,932918 & 0,87033599 & $-2,27999$ & 5,1983544 \\
\hline Messreihe 4 & 2,48672 & 6,18377636 & 1,37742 & 1,89728586 & 2,19989 & 4,83951601 \\
\hline Messreihe 5 & 0,051379 & 0,0026398 & $-0,0237296$ & 0,00056309 & $-2,30058$ & 5,29266834 \\
\hline Messreihe 6 & $-1,6817$ & 2,82811489 & 0,420397 & 0,17673364 & $-0,917509$ & 0,84182277 \\
\hline Summe & $-14,7903^{\circ}$ & $46,6789^{\circ} 2$ & $4,7691^{\circ}$ & $4,9917^{\circ} 2$ & $12,4965^{\circ}$ & $28,9176^{\circ} 2$ \\
\hline Mittelwert abs. & $2,1129^{\circ}$ & & $0,6813^{\circ}$ & & $1,7852^{\circ}$ & \\
\hline RMS & & $2,5823^{\circ}$ & & $0,8444^{\circ}$ & & $2,0325^{\circ}$ \\
\hline
\end{tabular}

Matching-Verfahren zu bilden. Die Abweichungen in der Position lassen sich über ein geeignetes Verfahren verringern. Welche Auswirkungen die Fehler in der Rotationsbestimmung haben, ist in weiteren Versuchen zu ermitteln.

Beispiel: Bei einer Abweichung des Kurswinkels $d \Psi$ von $4,6^{\circ}$ (siehe Tab. 2) ergibt sich bei einer Entfernung a von Kamera zum Objekt von etwa 50 m ein Fehler b der Geo-

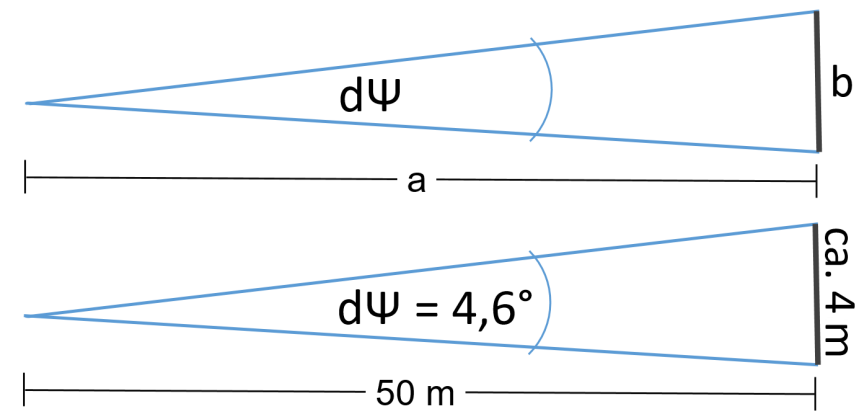

Abb. 6 Fehler bei der Georeferenzierung von $4 \mathrm{~m}$ bei einer Entfernung der Kamera zum Objekt von 50 m (Quelle: eigene Grafik).

referenzierung von ca. $4 \mathrm{~m}$ (siehe Abb. 6).

Beim späteren Matching-Verfahren kann es unter Umständen zu Fehlern kommen, wenn sich bspw. 2 ähnliche Muster innerhalb dieses Fehlers befinden. Das hängt jedoch immer von der jeweiligen Situation ab.

\section{Fazit}

Im Verlauf der Arbeit um die automatische Kalibrierung hat sich das implementierte Verfahren als Grundlage für weitere Entwicklungen und ernstzunehmender Bestandteil in der äußeren Orientierungsbestimmung erwiesen. sieren in diesem Zusammenhang auf Differential GPS (D-GPS) oder Echtzeitkinematik (RTK). Dadurch wird, auch für die Höhe, eine Positionsgenauigkeit im Zentimeterbereich ermöglicht.

In den Versuchen erreichte das System nicht die Genauigkeit des photogrammetrischen Verfahrens, stellt jedoch eine schnell einsetzbare Alternative dar. Besonders im mobilen und urbanen Bereich vereinfacht die automatische Kalibrierung den Einsatz solcher Systeme. In den weiteren Untersuchungen ist zu bestimmen, in wie weit die Abweichungen in Höhe und Kurswinkel das Matching-Verfahren beeinflussen.

\section{Literatur}

Flax B (2016) What Is the Update Rate on GPS Receivers? http:// www.ehow.com/info_12309199_update-rate-gps-receivers.html. Accessed 19 Feb 2016

Google (2016) Sky Map. https://play.google.com/store/apps/ details?id=com.google. android.stardroid\&hl=de. Accessed 21 Feb 2016

Grenzdörfer G, Zuev S (2007) Bestimmung des photogrammetrischen Genauigkeitspotentials des Online-Systems ANTAR zur luftgestützten Verkehrsdatenerfassung. In: Seyfert E (ed) Von der Medizintechnik bis zur Planetenforschung - Photogrammetrie und Fernerkundung für das 21. Jahrhundert. Dreiländertagung SGPBF, DGPF und OVG, Muttenz. Publikationen der DGPF, vol 16, pp 571-578

Kozempel K (2012) Entwicklung und Validierung eines Gesamtsystems zur Verkehrserfassung basierend auf Luftbildsequenzen. Dissertation, Deutsches Zentrum für Luft- und Raumfahrt, Berlin 
Leutron Vision (2013) PicSight-GigE user manual - Documentation for Leutron Vision GigE Vision cameras. http://www. leutron.com/index.php?elD=tx_nawsecuredl\&u=0\&file=fileadmin/download/privat/picsight/prot2010/manual/PicSightGigEManual. pdf\&t $=1376333749$ \&hash $=7 \mathrm{a}-$ 64ce637315c4787cc3022cc7652047689728fe. Accessed 05 Aug 2013

SkyTraq (2011) Application Note AN0003: Binary Messages of SkyTraq Venus 6 GPS Receiver. http://dlnmh9ip6v2uc. cloudfront.net/datasheets/Sensors/GPS/Venus/638/doc/ AN0003_v1.4.19.pdf. Accessed 05 Aug 2013

Wagenknecht D (2013) Erweiterung eines rechnergestützten Kamerasystems um die automatische Bestimmung der äußeren Einzelbildorientierung auf Basis von Navigationssensorik. Bachelorarbeit, Technische Hochschule Wildau; Deutsches Zentrum für Luft- und Raumfahrt, Berlin

\section{Autoren}

Sebastian Schulz

Deutsches Zentrum für Luft- und Raumfahrt e.V.

s.schulz@dlr.de

Daniel Wagenknecht

Deutsches Zentrum für Luft- und Raumfahrt e.V.

daniel.wagenknecht@gmail.com
(c) $9 \Theta$
Dieser Beitrag ist unter der Creative-Commons-Lizenz CC BY-NC-ND lizensiert. 